\title{
A Study of Prevalence and Most Common Genotypes of HPV (Human Papilloma Virus) by Molecular Methods and its Comparison to Pap smear, VIA and VILI Methods
}

\author{
R. Rajeswari ${ }^{*}$ \\ ${ }^{1}$ Assistant Professor, Department of I Microbiology, AIIMS, Udaipur
}

\author{
DOI: $10.36348 /$ sjpm.2021.v06i03.007 \\ | Received: 05.03.2021 | Accepted: 24.03.2021 | Published: 28.03.2021
}

*Corresponding author: Dr. R. Rajeswari

\section{Abstract}

Background: Infection with high-risk genotypes of Human papillomaviruses (HPV) is the major factor in the pathogenesis of cervical cancer. HPV genotypes 16,18,31,45 causes $90 \%$ of cervical cancer and these high-risk HPV genotypes cause nearly $100 \%$ of invasive cervical cancer. Cervical cancer can be prevented by vaccination against HPV high-risk genotypes. Effective cancer treatment programmes can improve survival rates for cervical cancer. Materials and Method: This study was aimed to evaluate the prevalence of HPV high-risk genotypes, so that high-risk individuals could be targeted, and specific genotypes associated with cervical cancer to be included in the vaccine preparations. Symptomatic patients visiting Gynaecology OPD of tertiary care hospital in India were recruited and Conventional PCR assay was done for detection of High-Risk genotypes of HPV causing cervical cancer. Statistical Analysis was done with PASW 18.0 (Predictive Analysis Software) by statistician. P value obtained by Chi Square test and p<0.05 was considered significant. Results: Out of the 72 symptomatic cases PCR was positive in 17 patients (23.6\%). In asymptomatic cases PCR positivity was $10.7 \%$. Out of the 100 patient's PCR was positive in $20 \%$ of the cases. Among the symptomatic PCR was positive in 17 cases. HPV -16 (9.7 \%) was the most common genotype among them. Infection with multiple genotypes 16 and 18 was positive in 6 cases $(8.3 \%)$, HPV -31 \&45 was positive in 2 cases $(2.8 \%$.) HPV 18 was positive in 2 cases (2.8\%) Among the asymptomatic HPV 16 in one case (3.5\%), HPV-31 in one case (3.5\%), HPV $-31 \& 45$ in one case $(3.5 \%)$. Conclusion: Our study results suggested inclusion of HPV 31 and 45 genotypes in $\mathrm{HPV}$ vaccine available and marketed in India.

Keywords: HPV-DNA analysis, PCR, HPV 31 and 45 genotypes, HPV Vaccines.

Copyright (C) 2021 The Author(s): This is an open-access article distributed under the terms of the Creative Commons Attribution 4.0 International License (CC BY-NC 4.0) which permits unrestricted use, distribution, and reproduction in any medium for non-commercial use provided the original author and source are credited.

\section{INTRODUCTION}

Infection with high-risk genotypes of Human papillomaviruses (HPV) is the major factor in the pathogenesis of cervical cancer [1]. It is preventable because it has long preinvasive stage which can be easily detected by mass screening methods. Cytology screening (Pap smear) is the standard diagnostic method for diagnosing cervical cancer at preinvasive stage itself.

HPV-DNA testing can be used in women before cervical abnormalities appear. According to CDC this test should be done only in women aged 30 to 65 years. Since HPV infections are common before 30 years which will be waste of money and unnecessary anxiety to women. PAP test and HPV-DNA co-testing can be used to increase screening interval to every 5 years.36 there are more than 100 genotypes of HPV. Among them 13 genotypes of HPV, HPV$16,18,31,33,35,39,45,51,52,56,5859,66$ are strongly associated with cervical cancer [2]. Among these highrisk genotypes, HPV genotypes 16,18,31,45 cause $90 \%$ of cervical cancer [3]. These high-risk HPV genotypes cause nearly $100 \%$ of invasive cervical cancer [2].

Cervical cancer can be prevented by vaccination against HPV high-risk genotypes. In 2006 first HPV vaccine was licensed for use in USA [4]. There are two HPV vaccines - Gardasil is a bivalent vaccine which contains HPV types 16 and 18. Cervarix is a quadrivalent vaccine which contains HPV genotypes 6,11,16,18.36 According to WHO vaccines against HPV in girls should be given at 9 to 13 years. Both vaccines can be given as 3 doses over a period of 6 months. 
HPV infected persons can also be vaccinated. Vaccination against HPV in girls combined with regular screening in women above 30 years can prevent precancerous lesions of cervix. Effective cancer treatment programmes can improve survival rates for cervical cancer [4].

The purpose of this study was to find out the high-risk individuals who might develop cervical cancer in future would be determined and to evaluate the prevalence of HPV high-risk genotypes so that high-risk individuals could be targeted and specific genotypes associated with cervical cancer to be included in the vaccine preparations.

\section{MATERIALS AND METHODS}

The present study was carried out in a Microbiology Department with Association of Gynaecology Department of a Medical College in Southern India for the duration of 1 year. Women of age group 20 to 65 years with history of white discharge, blood-stained discharge; heavy menstrual flow, intermenstrual spotting, and postcoital bleeding attending Gynaecology OPD were included in the study and pregnant women or women with history of hysterectomy were excluded. Prior permission from Institutional Ethical Committee and respective hospital authorities as well as written Informed Consent was obtained from the patients before enrolment in the study. Conventional PCR assay was done for detection of High-Risk genotypes of HPV causing cervical cancer.

For the extraction of DNA, the aliquots containing cervical biopsy were taken out of the deep freezer and phosphate buffer saline (PBS) was discarded. Tissue lysis buffer was added and kept for 10 minutes and the tissue was completely dissolved in the lysis buffer. It was then centrifuged, and pellet was formed the supernatant was discarded. The aliquots containing cervical scrapes were taken out of the deep freezer and brought to room temperature and then they were centrifuged $12000 \mathrm{rpm}$ for 10 minutes till pellet was formed. Freshly prepared PBS 140 microlitre was added to the pellet formed from cervical scrapings and biopsy and mixed well and 560microlitre of lysis buffer was added to that. Then 5.6 microlitre carrier RNA was added and then 20 microlitre of proteinase $\mathrm{K}$ was added. The contents were immediately mixed well and incubated at $56^{\circ} \mathrm{C}$ for 10 minutes and then 560 microlitre of $100 \%$ ethanol was added and mixed well by vortexing for 30 seconds. First 630 microlitre of sample was pipetted into pure fast spin column and it was then centrifuged at $12000 \mathrm{rpm}$ for one minute. Flow-through was discarded and the spin column was placed back into the same collection tube. The remaining 630 microlitre of sample was added and centrifuged for one minute. The flow-through was discarded and the spin column was placed back into the same collection tube. Wash Buffer-I, 500 microlitre was added to spin column and centrifuged at 12000rpm for $30-60$ seconds and the flow-through discarded. The spin column was placed into the same collection tube. Then 500 microlitre of wash Buffer-II was added to the spin column and centrifuged at 12000rpm for 60 seconds. The flow through was discarded and the spin column was placed back into the same collection tube. The empty spin column attached with collection tube was centrifuged at $12000 \mathrm{rpm}$ for an additional 3 minutes. This was done to remove residual ethanol and the collection tube was discarded. The spin column was transferred into a fresh $1.5 \mathrm{ml}$ micro centrifuge tube. Elution Buffer 60 microlitre was added to center of spins column membrane and incubated for 2 minutes at room temperature. It was then centrifuged at 12000rpm for one minute and spin column was discarded. The centrifuge tube contains the eluted nucleic acid.

After extracting purified DNA from cervical scrapings and cervical biopsy material, the DNA was amplified in a Thermocycler. Positive control for HPV16, 18, 31, 45 was used. Endogenous control Human GAPDH was used. The purpose of using Endogenous control was for a successful amplification of endogenous control indicates biological condition of the sample was suitable for diagnosis and integrity of the sample was maintained during storing. In all the samples Endogenous sample amplified well. Negative control used was Nuclease free water. The Master Mix used was $10 \mathrm{Mm}$ dntp MIX-0.5 $\mu 110$ x Taq Buffer-3 $\mu 1$ Taq Polymerase Enzyme-0.4 $\mu$ l Nuclease Free water14.1 10 X Red dye $-2 \mu 1$.

For the analysis by Agarose Gel Electrophoresis, $2 \%$ agarose gel prepared by, 2grams of agarose powder was mixed $100 \mathrm{ml}$ of distilled water and then heated in a microwave oven, mixed well until the Agarose was uniformly dissolved. After cooling to about $60{ }^{\circ} \mathrm{C}$ Ethidium bromide $5 \mu \mathrm{g} / \mathrm{ml}$ was added to facilitate visualization of DNA after electrophoresis. After cooling the solution, it was poured into casting tray containing a sample comb and allowed to solidify at room temperature. After the gel hardened enough, it was mounted in electrophoresis tank. Electrophoresis buffer was poured into the electrophoresis tank so that gel was completely immersed. The comb was carefully removed. Ethidium Bromide is mutagenic and should be handled with extreme caution.

The samples were delivered to the sample wells with a micro pipette. Negative control $10 \mu$ l was added in the first well. Positive control $10 \mu \mathrm{l}$ containing amplified HPV genotypes 16 and 18 were added in the second well. In the third well positive control containing $10 \mu \mathrm{l}$ of HPV -31 and 45 was added. DNA ladder $10 \mu \mathrm{l}$ was added in the fourth well. In the remaining wells amplified samples were added. A direct current (D.C) power source was connected to the 
electrophoresis apparatus and electrical current was set at $100 \mathrm{~V}$. As the amplified DNA has negative charge, it migrates from cathode (negative electrode) to anode (positive electrode). Run electrophoresis till the dye reaches three fourth distance of gel. The power pack was switched off and the gel tray was removed. The gel was placed into UV Transilluminator and observed for bands (to protect from UV exposure appropriate shield should be used).

Statistical Analysis was done with PASW 18.0 (Predictive Analysis Software) by statistician. P value obtained by Chi Square test and $\mathrm{p}<0.05$ was considered significant.

\section{RESULTS}

Among the 100 patients' women belonging to the reproductive age group were $90 \%$. Early age of marriage 17-18 years is associated with parity greater than three $(80 \%)$. Illiterate and women with only up to primary education were $76 \%$. Among the 100 patients $86 \%$ of the patients belonged to low socio-economic status. $88 \%$ of the women were non-vegetarians. Oral contraceptive use among the women was $6 \%$. Family history of cervical cancer among the 100 patients was 6 $\%$.

Among the 72 symptomatic patients white discharge was present in 71 patients $(98.6 \%)$, next symptom was menstrual irregularities in $12(16.7 \%)$, post coital bleeding in $5(6.9 \%)$, post-menopausal bleeding in $2(2.8 \%)$, inter menstrual spotting in 3 (4.2 $\%$.) patients.

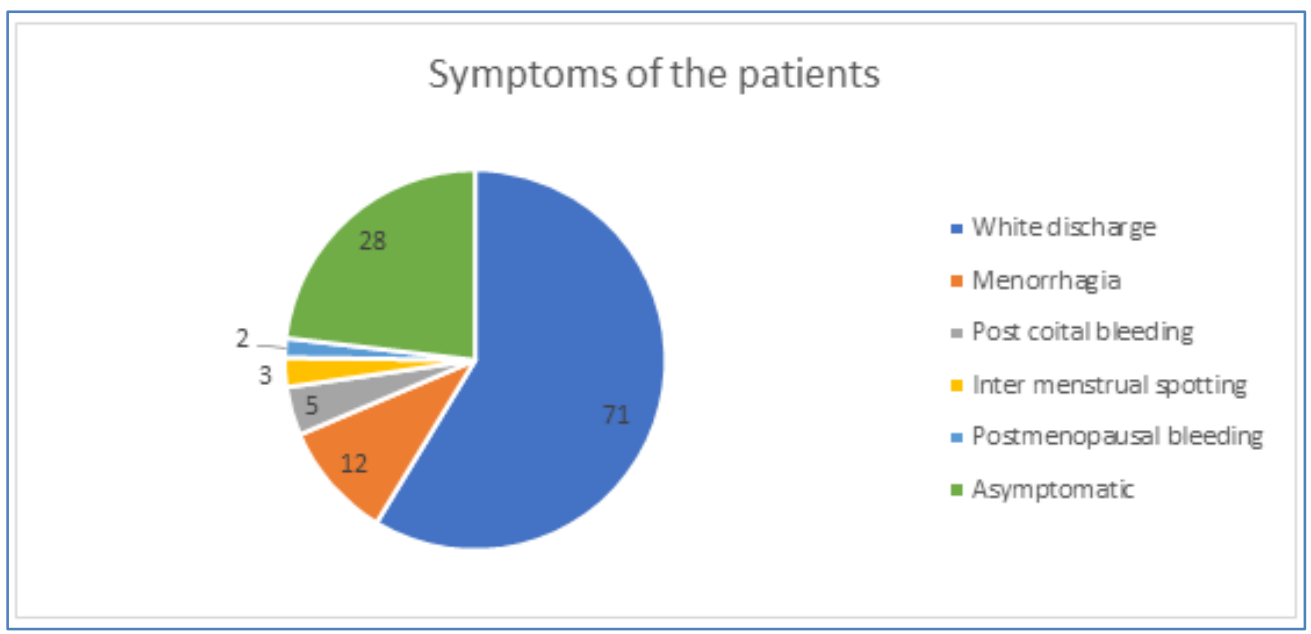

Fig-1: Symptoms of the patients

Among the hundred patients $84 \%$ had healthy cervix, 9\% had erosion and $7 \%$ had cervical growth. Among the 100 patients Pap smear report was normal study in $86 \%$, inflammatory smear in $7 \%$ and high grade squamous intraepithelial lesion in $7 \%$. The cervical biopsy report was Squamous cell carcinoma in $7 \%$ of the patients who had cervical growth. Chronic cervicitis was present in $7 \%$.

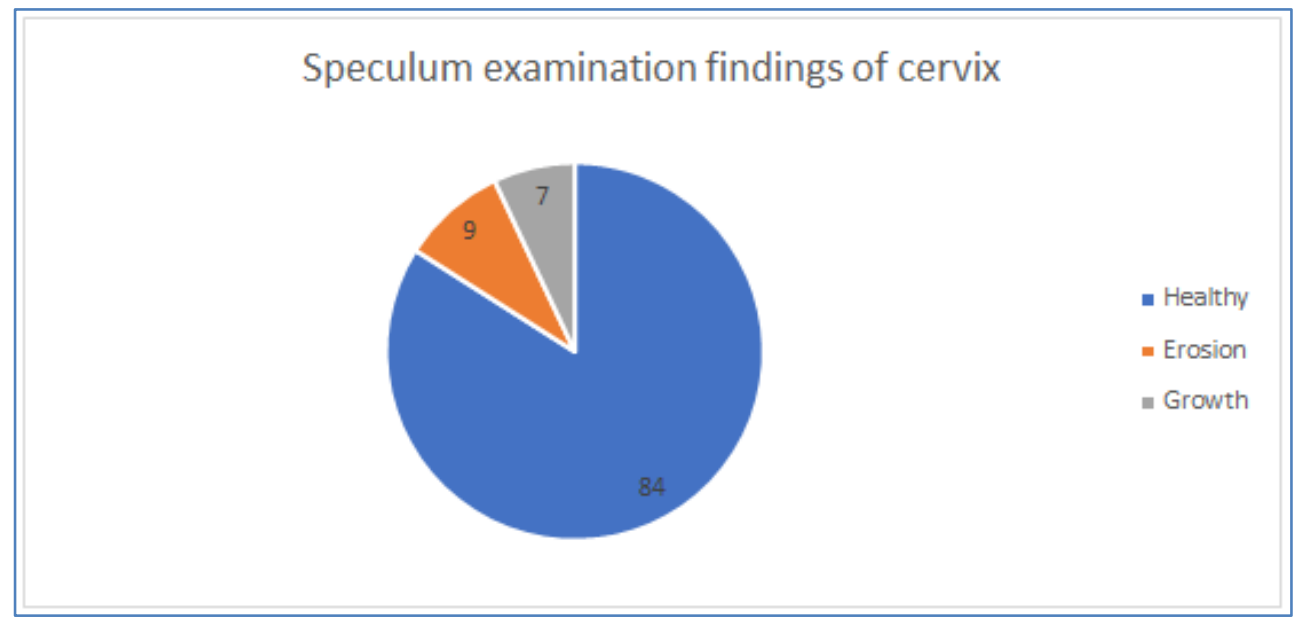

Fig-2: Speculum examination findings of cervix 
Out of the 72 symptomatic cases PCR was positive in 17 patients $(23.6 \%)$. In asymptomatic cases
PCR positivity was $10.7 \%$. Out of the 100 patient's PCR was positive in $20 \%$ of the cases.

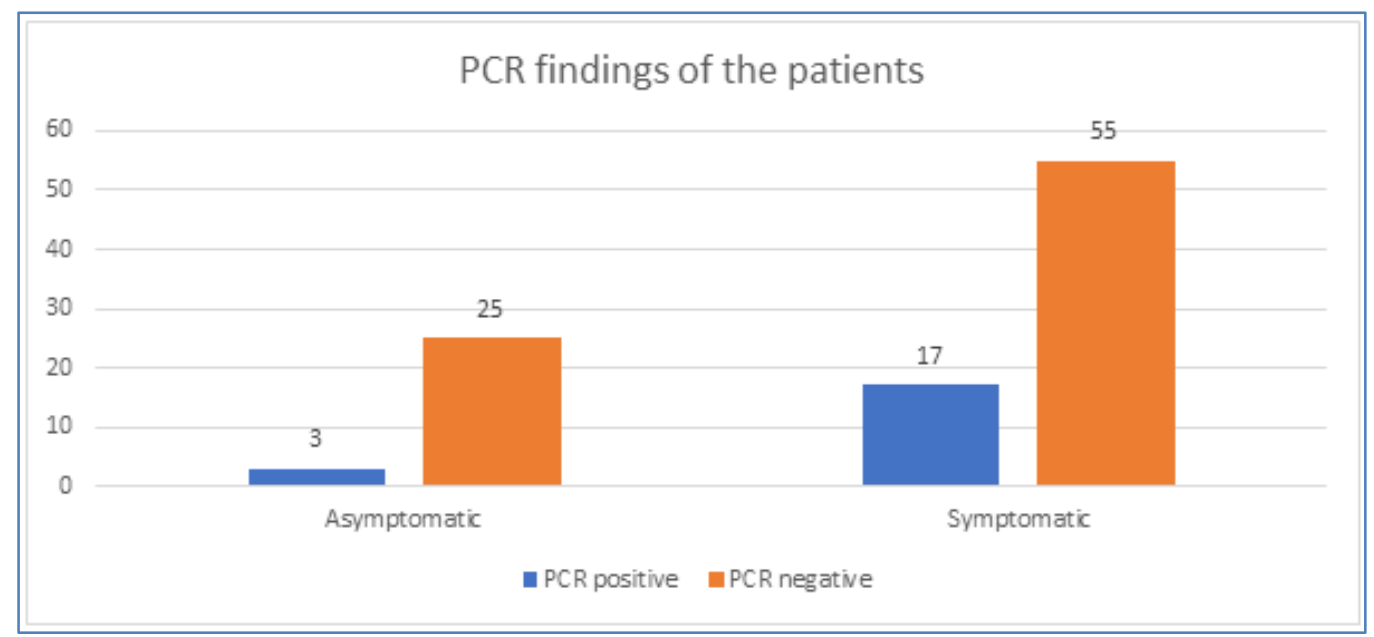

Fig-4: PCR findings of the patients

Among the 72 symptomatic cases 17 were positive for high-risk HPV genotypes. Among 17 HPV positive cases 7 had invasive cervical carcinoma of squamous cell carcinoma type, which was confirmed by Pap smear, cervical biopsy. The 7 cases were positive for HPV-16 and 18 by PCR. In the remaining 10 patients Pap smear, VIA|VILI was negative, but PCR was positive.
Among the 28 asymptomatic women 3 were positive for HPV high-risk genotypes. One patient was positive for HPV 16, another was positive for HPV 31 and last patient had multiple infections with HPV 31 and 45. In all the three patients Pap smear, VIA | VILI was negative. Total number of HPV infections among the 100 patients was 20. In that HPV-16 was the commonest type $(12.5 \%)$. Infection with Multiple genotypes 16 and 18 prevalence was $9.7 \%$. Prevalence of HPV 18 and 31, 45 was $2.8 \%$.

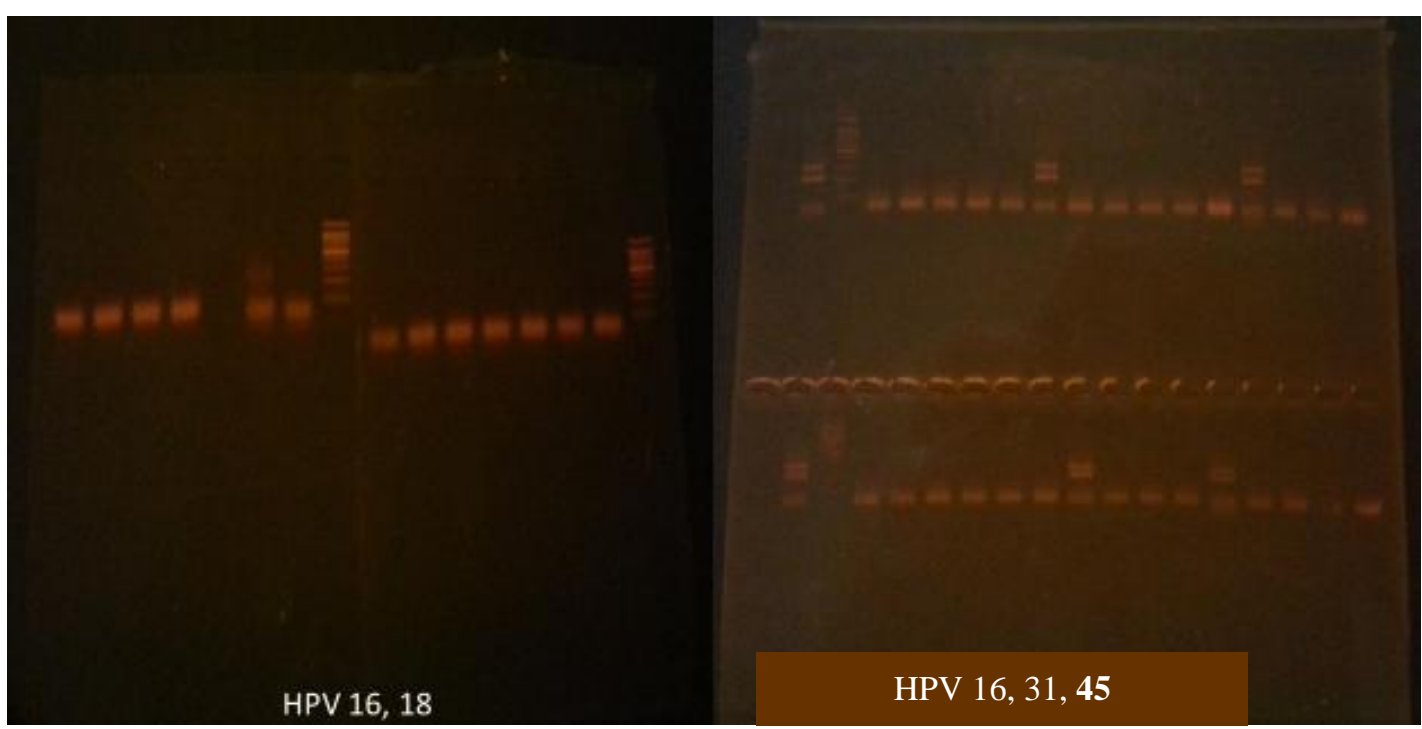

Fig-3: PCR Images obtained of HPV Genotypes 16,18,31,46 
Table-1: Genotype's category in the patients

\begin{tabular}{|c|c|c|c|c|c|}
\hline \multirow{2}{*}{\multicolumn{2}{|c|}{ Genotype Category }} & \multicolumn{2}{|c|}{$\begin{array}{c}\text { Number of women positive for HPV } \\
\text { DNA in asymptomatic }\end{array}$} & \multicolumn{2}{|c|}{$\begin{array}{c}\text { Number of women positive for HPV } \\
\text { DNA in symptomatic }\end{array}$} \\
\hline & & No & $\%$ & No & $\%$ \\
\hline \multirow{3}{*}{ I } & 16 & 1 & 3.5 & 7 & 9.7 \\
\hline & 18 & 0 & 0 & 2 & 2.8 \\
\hline & BOTH 16 and 18 & 0 & 0 & 6 & 8.3 \\
\hline \multirow{3}{*}{ II } & 31 & 1 & 3.5 & 0 & 0 \\
\hline & 45 & 0 & 0 & 0 & 0 \\
\hline & BOTH 31 and 45 & 1 & 3.5 & 2 & 2.8 \\
\hline & Total & \multicolumn{2}{|c|}{3} & \multicolumn{2}{|c|}{17} \\
\hline
\end{tabular}

\section{DISCUSSIONS}

In the present study among the 72 symptomatic patients white discharge was present in 71 patients $(98.6 \%)$, next symptom was menstrual irregularities in $12(16.7 \%)$, post coital bleeding in 5 $(6.9 \%)$, post-menopausal bleeding in $2(2.8 \%)$, inter menstrual spotting in $3(4.2 \%$.) patients. The use of oral contraception for less than two years in our study was 6 $\%$. A study conducted by Maria G Centurion et al. [5] was $27 \%$. According to WHO [6], women who took OCP greater than 5 years had $51 \%$ increased risk and $123 \%$ risk if taken for more than 8 years.

Family history of cervical cancer in our study was 4\%. According to Cancer Research UK [7] a woman has double the risk if she has first degree relative (mother, sister, daughter) diagnosed with Adenocarcinoma or Squamous cell carcinoma. The reason for the risk may be faulty gene, or having same lifestyle pattern, or HPV infection passed on during pregnancy or childbirth.

In our study on speculum examination cervix was healthy in $84 \%$, erosion in $9 \%$ patients, growth in $7 \%$, patients. Pap smear report in our study is normal study in $86 \%$, inflammatory smear in $7 \%$, neoplastic changes in $7 \%$ patients. Cervical biopsy was done. It showed chronic cervicitis in $9 \%$ of patients, and Squamous cell carcinoma in $9 \%$ patients.

Conventional PCR for high-risk HPV genotypes $16,18,31,45$ was done. PCR was positive in $23.6 \%$ of the symptomatic patients. This correlates with study conducted by Giftson J Senapathy et al. [8] in which it was $21.7 \%$. In this study out of 28 asymptomatic women screened $10.7 \%$ are associated with HR-HPV types which correlate with the study done in India by Shiksha Srivastava et al. [9] (9.9\%). Infection with HPV 16 and 31 and 45 is $3.5 \%$. The study done by Jacobs et al. [10] showed $8.6 \%$. Among symptomatic, the most common genotype is HPV-16 (9.7 \%), HPV-18 2.8\%. Multiple genotypes like 16 and 18 is $8.3 \%$. HPV -31 and 45 is $2.8 \%$. HPV -16 infections in symptomatic women is $9.7 \%$. This correlated with the study done by Rosita Verteramo et al. [11] in which it was most prevalent and was about $14.5 \%$. The study done by IARC surveys in Asia and Africa is $14 \%$. Patients with multiple genotypes 16 and 18 is $8.3 \%$. The study done by Si-Mohamed et al. [12] was $20.7 \%$. This shows that in our study it was lower. In our study infection with multiple genotypes 31 and 45 is $2.8 \%$. This correlates with study done by Partha Basu [13] where it was $4.7 \%$.

\section{CONCLUSION}

This study shows the importance of inclusion of HPV 31 and 45 genotypes in HPV vaccine.

\section{ACKNOWLEDGEMENT}

We are thankful to administration of our hospital and all the supporting staff including doctors, nurses and non-medical personnel for helping us at different stages during the conduct of the study. We are grateful of all our patients for proving consent to participate in this study.

\section{REFERENCES}

1. Sowjanya, A. P., Jain, M., Poli, U. R., Padma, S., Das, M., Shah, K. V., \& Ramakrishna, G. (2005). Prevalence and distribution of high-risk human papilloma virus (HPV) types in invasive squamous cell carcinoma of the cervix and in normal women in Andhra Pradesh, India. BMC infectious diseases, 5(1), 1-7.

2. Haghshenas, M., Golini-Moghaddam, T., Rafiei, A., Emadeian, O., Shykhpour, A., \& Ashrafi, G. H. (2013). Prevalence and type distribution of highrisk human papillomavirus in patients with cervical cancer: a population-based study. Infectious agents and cancer, 8(1), 1-6.

3. Gomez, D. T., \& Santos, J. L. (2007). Human papillomavirus infection and cervical cancer: pathogenesis and epidemiology. Communicating current research and educational topics and trends in applied microbiology, 1, 680-8.

4. Haverkos, H. W. (2005). Multifactorial etiology of cervical cancer: a hypothesis. Medscape general medicine, 7(4), 57.

5. Centurioni, M. G., Puppo, A., Merlo, D. F., Pasciucco, G., Cusimano, E. R., Sirito, R., \& Gustavino, C. A. (2005). Prevalence of human papillomavirus cervical infection in an Italian 
asymptomatic population. BMC Infectious diseases, 5(1), 1-7.

6. Comprehensive cervical cancer prevention and control. (2013). A healthier future for girls and women - WHO GUIDANCE NOTE - 2013.

7. Cancer Research UK - About Cervical Cancer, a Quick Guide.

8. Senapathy, J. G., Umadevi, P., \& Kannika, P. S. (2011). The present scenario of cervical cancer control and HPV epidemiology in India: an outline. Asian Pac J Cancer Prev, 12(5), 11071115 .

9. Srivastava, S., Gupta, S., \& Roy, J. K. (2012). High prevalence of oncogenic HPV-16 in cervical smears of asymptomatic women of eastern Uttar Pradesh, India: a population-based study. Journal of biosciences, 37(1), 63-72.

10. Jacobs, M. V., Snijders, P. J., Voorhorst, F. J., Dillner, J., Forslund, O., Johansson, B., \& Walboomers, J. M. (1999). Reliable high risk HPV DNA testing by polymerase chain reaction: an intermethod and intramethod comparison. Journal of clinical pathology, 52(7), 498-503.

11. Verteramo, R., Pierangeli, A., Mancini, E., Calzolari, E., Bucci, M., Osborn, J., \& Degener, A. M. (2009). Human Papillomaviruses and genital co-infections in gynaecological outpatients. $B M C$ Infectious Diseases, 9(1), 1-7.

12. Si-Mohamed, A., Kazatchkine, M. D., Heard, I., Goujon, C., Prazuck, T., Aymard, G., \& Bélec, L. (2000). Selection of drug-resistant variants in the female genital tract of human immunodeficiency virus type 1 -infected women receiving antiretroviral therapy. The Journal of infectious diseases, 182(1), 112-122.

13. Basu, P., Ponti, A., Anttila, A., Ronco, G., Senore, C., Vale, D. B., \& Sankaranarayanan, R. (2018). Status of implementation and organization of cancer screening in The European Union Member States-Summary results from the second European screening report. International journal of cancer, 142(1), 44-56. 